\title{
Statistical Learning Ability as a Measure of Cognitive Function
}

\author{
Steffen A. Herff (steffen_herff@ihpc.a-star.edu.sg) \\ ${ }^{1}$ Social and Cognitive Computing Department, Institute of High Performance Computing \\ A*STAR, 1 Fusionopolis Way, Singapore 138632; \\ ${ }^{2}$ MARCS Institute for Brain, Behaviour and Development, Western Sydney University, Australia \\ Nur Amirah Abdul Rashid (nur_amirah_abdul_rashid@imh.com.sg) \\ Research Division, Institute of Mental Health, 10 Buangkok View, \\ Singapore 539747
}

\author{
Jimmy Lee (jimmy_lee@imh.com.sg) \\ North Region \& Research Division, \\ Institute of Mental Health, 10 Buangkok View, \\ Singapore 539747
}

Tih-Shih Lee (tihshih.lee@duke-nus.edu.sg)

Neuroscience and Behavioral Disorders Programme, Duke-NUS Graduate Medical School, Singapore, Singapore.

\author{
Kat R. Agres ${ }^{1,2}$ (kat_agres@ihpc.a-star.edu.sg; muskra@nus.edu.sg) \\ ${ }^{1}$ Social and Cognitive Computing Department, Institute of High Performance Computing \\ A*STAR, 1 Fusionopolis Way, Singapore 138632; \\ ${ }^{2}$ National University of Singapore, 3 Conservatory Drive, Singapore 117376
}

\begin{abstract}
Statistical Learning (SL), the ability to extract probabilistic information from the environment, is a subject of much debate. It appears intuitive that such a profound mechanism of learning should carry predictive power towards general cognitive ability. Yet, previous attempts have struggled to link SL ability to measures of general cognitive function, suffering from low correlations and mediocre test-retest reliability. Here, we deploy a new continuous auditory SL task that achieves high test-retest reliability $(\sim r=.8)$ and shows that SL ability does significantly correlate with general cognitive function (up to $r$ $=.56$ ). Results are discussed in light of i) the theoretical implications of the high test-retest reliability of our novel SL task, ii) SL ability as a marker of general cognitive function, and iii) future methodological considerations.
\end{abstract}

Keywords: Statistical Learning; Cognitive Assessment; Auditory Domain; Continuous Paradigm; Elderly

\section{Introduction}

Statistical learning (SL) is the ability to track probabilistic dependencies in the environment and make predictions based on such probabilities. The importance of SL has been shown in language acquisition, where humans are able to learn segment boundaries between words based on the transitional probabilities of speech sounds (Saffran, Aslin, \& Newport, 1996)(see Saffran \& Kirkham, 2018 for a review). SL is not only involved in language acquisition but also plays a major role in any probabilistic-based build-up of expectancies regardless of the domain, such as music (Creel, Newport, \& Aslin, 2004; Moldwin, Schwartz, \& Sussman, 2017) and vision (Kirkham, Slemmer, \& Johnson, 2002, see Erickson \& Thiessen, 2015 for a review). Considering that SL is a profound mechanism of learning, it comes by no surprise that much research has been investigated the use of SL paradigms as a measurement of individual ability and cognitive assessment (Kaufman et al., 2010; Siegelman, Bogaerts, Christiansen, \& Frost, 2017; Siegelman \& Frost, 2015; Unsworth \& Engle, 2005).

Traditionally, most assessments of cognitive function are based on a wide battery of tests, ranging from various memory tasks to verbal fluency tests and visual decisionmaking tasks. These tests tend to be combined into a summary statistic that is then interpreted to reflect the overarching cognitive capacity of an individual (e.g., BACSF in Keefe et al., 2004; Lam et al., 2017). SL paradigms may offer a possible unified alternative measure of general cognitive capacity, because of their ability to capture an essential mechanism of learning involved in perception as well as behavior (Kaufman et al., 2010).

Previous research investigating a connection between SL ability and general cognitive ability, however, has often yielded inconclusive results, with only small correlations detected (e.g., $r$ between 0.019 and .19 in Feldman, Kerr, and Streissguth, 1995). Kaufman et al. (2010) used a set of probabilistic Serial Reaction Time tasks to investigate the connection between implicit SL and general cognitive ability. In this paradigm, a sequence of states instantiated by a stimulus at four different locations on the screen was presented. The probability of each state's occurrence in the 
sequence was controlled by an underlying transitional probability matrix. By extracting the statistical regularities of the underlying transitional probability matrix, participants could build expectations that would facilitate their reaction times. The authors deliberately chose a reaction time task to measure learning on a trial-by-trial basis. This is an important consideration because learning curves may provide valuable information about individual cognitive capacities (Karuza et al., 2013; Misyak, Christiansen, \& Tomblin, 2010). Similar to previous attempts, however, the implicit SL ability in this study showed small correlations with general cognitive ability $(r=.16)$. A reason for this may lie in the implicit nature of the deployed task.

It is important to note that the paradigm used by Kaufman et al. (2010) was aiming to maximize incidental learning without highlighting the probabilistic nature of the task. The degree of implicit vs. explicit information participants are provided with impacts SL performance (Seger, 1994). With explicit instructions, participants are aware that there exists an underlying probabilistic structure for them to learn, whereas in an implicit task that insight must also has to be extracted first. In general, it seems that explicit instructions increase the predictive power of SL performance for general cognitive ability (Unsworth \& Engle, 2005). Here, we aim to deploy an explicit SL paradigm that is still able to capture continuous learning trajectories.

Further methodological insight comes from Siegelman, Bogaerts, and Frost (2017) who analyzed existing SL paradigms to identify their shortcomings for the purpose of measuring individual SL ability. One of the key criticisms was that many participants perform at chance level (21-47\%), so SL can often only be demonstrated at a group level. The authors also observed that many SL paradigms deploy only a small number of different items (mostly triplets with 4 to 8 different items) that are repeated multiple times ( 1 to 16 times). A result of this approach is that there is little to no variability of difficulty between the items, resulting in little information to precisely estimate individual SL ability. Another concern is that most SL paradigms show low testretest reliability $(r=.44$ in Kaufman et al., 2010). Though Siegelman, Bogaerts, and Frost (2017) did not aim to link SL ability to general cognitive ability, these are important considerations, as a precise measurement of SL ability is a prerequisite to establish SL ability as a predictor of general cognitive function.

Based on their criticism of existing paradigms, Siegelman, Bogaerts, and Frost (2017) developed a new Visual SL paradigm that provides a promising measure of individual SL ability. However, in this new paradigm, the authors rely on the traditional learning-test phase distinction. This separation between learning and testing can influence participants' responses, introducing noise into the outcome measure and does not allow tracking of continuous learning. Here, we aim to combine Siegelman et al's (2017) criticism of commonly used SL paradigms with Kaufman et al. (2010) continuous measurement approach. To this end, in the present study, we test a continuous paradigm with a unique stimulus in each trial, only defined by its transitional probabilities.

Importantly, while Siegelman, Bogaerts, and Frost's (2017) new paradigm is conducted in the visual domain, here we aim to explore the relationship between general cognitive ability and auditory SL. Given the link with language acquisition in the early years of development (Saffran \& Kirkham, 2018), auditory stimuli have been widely used in SL paradigms. The auditory domain is a promising target to measure SL ability and link it to cognitive ability. This is because the auditory domain specializes in processing stimuli that unfold in time (Pérez-González \& Malmierca, 2014) and relies heavily on extracting statistical information from the environment (Agres, Abdallah, \& Pearce, 2018). Indeed, the auditory system is very proficient in extracting probabilistic regularities (Agres, Abdallah, \& Pearce, 2018; Barascud, Pearce, Griffiths, Friston, \& Chait, 2016) and appears to outperform other modalities in terms of SL (Conway \& Christiansen, 2005). Furthermore, the plethora of emerging neural correlates of auditory SL can provide a useful resource in the future to understand the link between SL ability and general cognitive function (Abla, Katahira, \& Okanoya, 2008; Barascud et al., 2016; Moldwin et al., 2017).

Unfortunately, previous attempts to connect auditory SL to general cognitive ability have been futile (Siegelman \& Frost, 2015), yielding non-significant correlations between -.06 and 0.06 . These findings provide preliminary support for the null hypothesis of no significant connection between SL ability and general cognitive ability. Yet, there is still much debate about whether SL ability is domain dependent, or subject to a shared underlying computation mechanism that may or may not be related to general cognitive ability (Conway \& Christiansen, 2005; Conway \& Pisoni, 2008; Frost, Armstrong, Siegelman, \& Christiansen, 2015; Siegelman \& Frost, 2015). For example, Frost, Siegelman, Narkiss, and Afek (2013) demonstrated that a performance on a SL task correlated with various language learning scores (up to $r=$ .57). Similar results have been reported by Ahissar, Lubin, Putter-Katz, and Banai (2006), who found that dyslexia may be related to difficulties with tracking and extracting statistical regularities. As a result, it has been suggested that there is a universal principle of SL that functions domain agnostically (Frost et al., 2013).

Further research is required to explore this possible relationship between SL ability and general cognitive function. The present study is in an attempt to link SL ability to cognition through a refined paradigm that draws from recent methodological advances in the literature. Demonstrating that SL ability holds predictive value for traditional measurements of cognitive function would be a first step towards a new practical measurement of cognitive ability that may function domain agnostically and would not require an entire cognitive test battery with a diverse set of tasks to be measured effectively. Furthermore, such a finding would support the suggestion that SL is a unified principle that provides insight in fundamental cognitive processes (Ahissar et al., 2006; Frost et al., 2013). 
We aim here to explore i) test-retest reliability of a novel SL paradigm, and ii) whether this paradigm may be used to approximate cognitive ability as measured by a conventional, well established tool. Importantly, we also hope to iii) gain insight into statistical learning performance that will help in refining and further developing the capacity of SL tasks to capture general cognitive ability and deepen our understanding of statistical learning at large. The analysis and discussion below address each of these three aims in turn.

\section{Methodology}

\section{Participants}

The present investigation uses a subset of a large clinical music BCI study that is part of a collaboration between [intentionally left blank for blinded review]. The present work focuses on an SL task and cognitive assessment data. The remaining data from the larger project will be reported elsewhere. Participants in the clinical BCI study were between 60 and 85 years old, English speaking, Geriatric Depression Scale (GDS) score of 4 or below, fit to provide informed consent, and able to travel to the study site independently. Exclusion criteria were the presence of hearing impairment, presence of any known neuropsychiatric disorder (such as dementia, or epilepsy), or any contraindications for MRI. Participation in the entire study was reimbursed with $\mathrm{S} \$ 100$. A total of 30 participants participated in the study, however, three participants were removed from the sample due to incomplete datasets. The age of the remaining 27 participants ranged from 62 to $81\left(M_{\text {age }}\right.$ $=67.7, S D_{\text {age }}=5.1$ ).

\section{Procedure}

Participants provided informed consent before completing the questionnaires and assessments. Participants completed the Brief Assessment of Cognition - Short Form (BAC-SF), which consists of the Verbal Memory, Digit Sequencing and Symbol Coding tasks, and takes approximately 15 minutes to complete (Keefe et al., 2004; Lam et al., 2017). Data from these three tasks were normed (Lam et al., 2014). Gender/age adjusted z-scores were obtained via the PCA method, as detailed in Lam et al. (2017). Cognitive assessment was administered before the SL task. At the end of the clinical trial the cognitive assessment ( 9 to 10 weeks later), as well as the statistical learning task ( 8 to 9 weeks later)were administered again. In the following section, the SL task is described in detail.

A Novel Paradigm We developed and deployed a novel statistical learning paradigm. Traditional SL paradigms consist of an exposure and a testing phase. While such paradigms allow assessment of SL ability at the end of the experiment, they do not provide insight into the information acquisition rate or the learning trajectory. Notably, however, there can be large differences in the learning curves of individuals that may provide valuable information about individual cognitive capacity (Karuza et al., 2013; Misyak et al., 2010). The current SL paradigm consists of a continuous task, rather than being divided in an exposure and a testing phase. Participants were presented with a continuous stream of four distinguishable states (A, B, C, D). These states were instantiated by tones (sine waves at $\sim 165 \mathrm{~Hz}$ (E3), $220 \mathrm{~Hz}$ (A3), 294 Hz (D4), 392 Hz (G4), each 333ms in duration), presented through Sennheiser HD 201 headphones. Each sound was uniquely associated with one state. The stream of tones could randomly stop between 3.6 to $5.3 \mathrm{secs}$ (11 to 17 states), and the participant's task was to indicate which state they suspect will occur next. They responded by clicking one of four buttons that were presented on the computer screen. Each button was associated with one of the four states. After their response, the stream continued. Unbeknownst to the participants, the transitional probability matrix between the four states was tightly controlled. As visualized in Figure 1, the transitional probability matrix yields three different pathways: A high probability pathway that reflects responses that are concurrent with the most likely next state; a low probability pathway that reflects participants' responses with one of the two less likely states; and a rule violation. Rule violations reflect responses that indicate a repetition of a state. Such repetitions are impossible within the given transitional probability matrix. There is a plethora of possible measurements that can be calculated based on participants' responses to this paradigm. Here, we use two fundamental summary statistics that capture overall performance whilst simultaneously capturing aspects of the individual's learning trajectory.

Cumulative High Probability Choices (CHPC) responses reflect the cumulative number of responses that lie on the high probability pathway. In the beginning of the task, such responses should occur with chance probability $(25 \%)$; however, as soon as the participant extracts information from the transitional probability matrix, the rate in which these responses occur should increase. At the end of the task, CHPC provides a summary of overall performance and takes learning rate into account.

Cumulative Rule Violations (CRV) reflect the number of responses that indicate repetition, which are impossible in the transitional probability matrix. In the beginning of the task, rule violations should occur with chance probability $(25 \%)$; however, the rate in which rule violations occur should rapidly decrease. Therefore, by the end of the task, CRV should provide a summary of performance whilst also taking learning rate into account. 


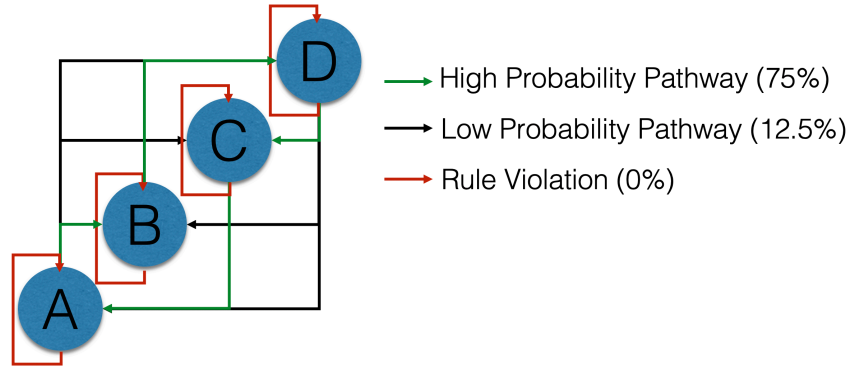

Figure 1: Schematic representation of the probability pathways. Each state is most often ( $75 \%$, green line) followed by a specific other state. Repetition of the same state is impossible $0 \%$, red line). The remaining two other states are equiprobable ( $12.5 \%$ black line)

All instructions were displayed on a computer monitor. To ensure that participants understood the task, each participant completed four short practice phases. The first phase consisted of a short demonstration of the four possible states in ascending and descending order. In the second phase, participants had the opportunity to familiarize themselves with the auditory states and the interface. Participants heard the four different states, one at a time in random order, and were asked to indicate the correct button that was associated with the respective state. The phase was complete when the participant achieved 6 out of the last 10 trials correct, or completed a total of 20 trials. In the third phase, participants were presented with four trials of deterministic (rather than probabilistic) sequences. When each sequence stopped, the participant was required to indicate the state that they believed was most likely to come next. In this training phase, the associated buttons flashed simultaneously as the sounds were played. This effectively reduced task difficulty by making the sequences multimodal. The fourth phase consisted of two trials and was identical to the third phase, except that the buttons did not flash, so the participant had to rely solely on auditory information. Afterwards, participants continued on to the main study, which consisted of one long sequence drawn from the transition probability matrix shown in Figure 1. Similar to practice phase four, the states were purely instantiated auditorily. In total, the long stream was interrupted 48 times (trials) to prompt the participant to predict the next state using the visual interface.

\section{Results}

Similar to (Kaufman et al., 2010), we use correlations to explore test-retest reliability, and the link between SL and Cognitive ability. To investigate SL performance, we deploy a simulation-based approach to identify chance as well as ideal performance in this probabilistic paradigm.

i) Test-retest Reliability As seen in Table 1, the adjusted zcomposite scores of the BAC-SF cognitive assessment ( $r=$ $.90, t(25)=10.515, p<.0001)$ as well both statistical learning measurements -- CHPC $(r=.84, t(25)=7.70, p<.0001)$ and $\mathrm{CRV}(r=.79, t(25)=6.44, p<.0001)--$ show strong and significant correlations between the first and second visit. Bonferroni corrected (new $\alpha=0.0166$ ) paired sampled t-tests of CHPC between the first $(M=14.85, S D=7.22)$ and second $(M=15.48, S D=7.65)$ visit revealed no significance changes $(t(26)=-0.77, p=0.4477)$. There was also no significant difference in CRV $(t(26)=-0.06, p=0.9541)$ between the first $(M=8.52, S D=4.66)$ and second $(M=8.56, S D=5.35)$ visit. After Bonferroni correction, there were also no significant differences $(t(26)=-2.27, p=0.0319)$ in the adjusted z-composite scores of the BAC-SF cognitive assessment between the first $(M=0.23, S D=1.12)$ and second $(M=0.45, S D=1.15)$ visit. Taken together, the results indicate high test-retest reliability for all three measurements.

Table 1: Correlations between age and gender adjusted zcomposite scores for the BAC-SF cognitive assessment (Comp), and both measures of statistical learning (CHPC, CRV) across both visits (V1, V2)

\begin{tabular}{lllllll}
\hline & CHPC & CRV & Comp & CHPC & CRV & Comp \\
& V1 & V1 & V1 & V2 & V2 & V2 \\
\hline V1:CHPC & 1 & & & & & \\
V1:CRV & -.80 & 1 & & & & \\
V1:Comp & .48 & -.40 & 1 & & & \\
V2:CHPC & .84 & -.80 & .54 & 1 & & \\
V2:CRV & -.67 & .79 & -.35 & -.70 & 1 & \\
V2:Comp & .52 & -.44 & .90 & .56 & -.44 & 1 \\
\hline
\end{tabular}

ii) Statistical Learning and Cognitive Ability Across both visits, the adjusted $\mathrm{z}$-composite scores of the BAC-SF cognitive assessment show moderate and significant correlation with CHPC $(r=0.52, t(52)=4.44, p<0.0001)$ and CRV $(r=-0.42, t(52)=-3.31, p=0.0017)$; see Table 1 for the correlations across visits. A linear model predicting adjusted z-composite scores of the BAC-SF cognitive assessment using CHPC, CRV, Visit, and their interactions as predictors revealed significant coefficients for CHPC (Est $=0.12, S D=0.05, p=0.0181)$, and a significant interaction between CHPC x CRV ( $E s t=-0.01, S D=0.01, p=0.0389$ ). No other main effects or interactions reached significance (all $p>0.1402$ ). This suggests that CHPC and CRV share considerable information, an observation supported by the strong overall correlation for both visits between CHPC and CRV $(r=-0.74, t(52)=7.96, p<0.0001)$; see Table 1 for the correlations across visits. In direct comparison, however, CHPC is the better predictor of the adjusted z-composite scores for the BAC-SF cognitive assessment compared with CRV. Figure 2 shows a marginal effect plot for the significant effect of CHPC on the composite score across both visits and CRV at its average. The CRV however, shapes CHPC's prediction, as revealed by the significant and negative CHPC $x$ CRV interaction term. This is also visualized in the marginal effect plots seen in Figure 2 that combine data from both visits. 


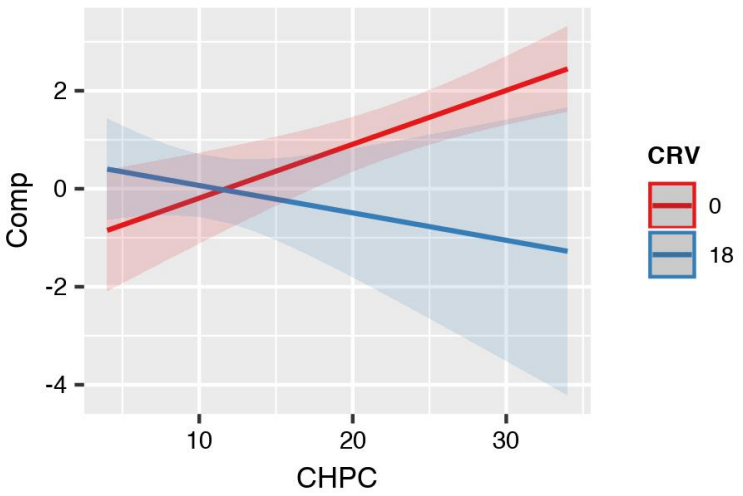

Figure 2: Marginal effects of the CHPC x CRV interaction on age and gender adjusted z-composite scores of the BAC-SF cognitive assessment (Comp). The bands show a $95 \% \mathrm{CI}$ around the prediction line. The figure shows that increasing CHPC predicts high cognitive ability when participants make few rule violations (CRV red line). This, however, is negated for participants who make many rule violations (CRV blue line).

iii) Statistical Learning Performance. Because this is a probabilistic rather than deterministic task, the question of what constitutes chance, and what constitutes ideal performance, is non-trivial and has to be carefully considered. To model ideal and chance performance, we simulated 10,000 observers providing random responses ( $25 \%$ for each state) and constructed a $95 \% \mathrm{CI}$ around the resulting CHPC. This probability band is displayed towards the bottom in Figure 3, and participants that fall inside of this band are considered to be performing at chance level. Furthermore, we simulated 10,000 ideal Bayesian observers with a weak prior towards providing equiprobable responses. The beta distribution is updated after every state during each trial. The resulting beta distribution at the end of each trial was used to draw an ideal observer response to determine whether the observer produces responses that follow the high probably pathway. The grey band towards the top of Figure 3 shows a $95 \%$ CI around the response distributions of the ideal Bayesian observer. This band can be considered the best achievable performance. We conducted the same simulation for CRV responses.

Using the CHPC metric, 7 participants (out of 27) on the first and 8 participants on the second visit successfully learned the high probability pathway. In terms of rule violations, 7 participants on the first, and 11 participants on the second visit successfully learned to avoid repetitions. Every participant that performed above chance on the first visit also did so during the second. Furthermore, CHPC and CRV are strongly negatively and significantly correlated across both visits $(r=-0.74, t(52)=-7.96, p<0.0001)$; see Table 1 for the breakdown of correlations across visits.

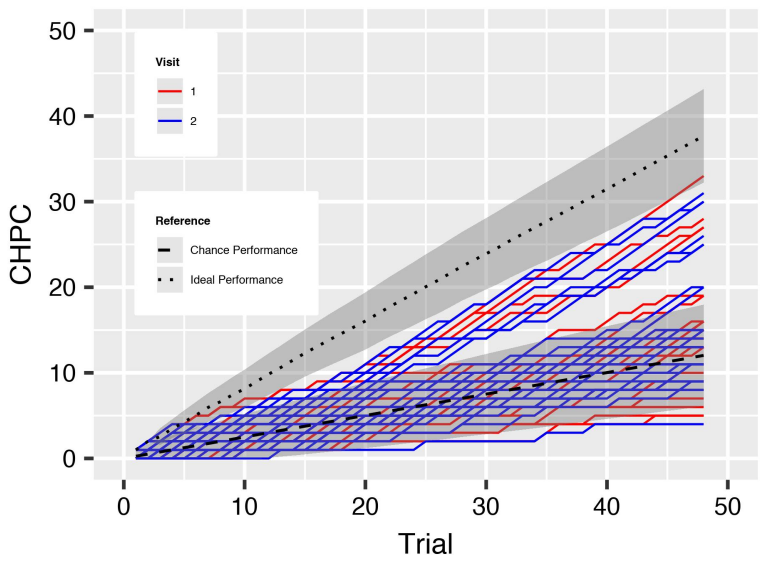

Figure 3: CHPC performance for all 27 participants for both visits. The dashed line represents chance performance and the dotted line performance of an ideal Bayesian observer. Grey bands represent $95 \%$ CIs.

\section{Discussion}

The present study investigated a possible link between SL ability and general cognitive function. Indeed, using our new SL paradigm, such a connection was observed. The results above are discussed below in terms of test-retest reliability, $S L$ and cognitive ability, as well as CHPC and CRV Performance.

Test-retest Reliability The BAC-SF cognitive assessment showed high test-retest reliability between the two sessions $(r=.90)$. This is a minimum prerequisite for any cognitive assessment used within a clinical context, and lends credibility to the measurement. In this particular case, the high test-retest reliability is worth noting, as the BAC-SF (Lam et al., 2014; Lam et al., 2017) is only a short form of the BACS (Keefe et al., 2004). Previous research investigating test-retest reliability of SL measurements has only yielded moderate test-retest reliability $(r=.44$ to $r=.58$, in Kaufman et al., 2010, and Siegelman \& Frost, 2015). These values are low compared to the typical test-retest reliability in psychological cognitive assessments measurements $(\sim r=$ .8 see Nunnally \& Bernstein, 1994). Indeed, even Siegelman, Bogaerts, and Frost's (2017) new improved visual SL task falls slightly short of this target with a test-retest reliability of .68. The measurements deployed here, achieve high testretest reliability ( $r=.79$ to .84$)$, and thereby approach the range commonly observed in psychological assessments. The This provides support for the view that SL is a stable individual ability, which is necessary for a test to function as a measurement of cognitive ability (see Siegelman \& Frost, 2015 for a discussion).

Statistical Learning and Cognitive Ability Both measurements of SL ability carry meaningful predictive information for cognitive ability. High CHPC, which indicates successful SL performance, has significant positive 
correlations with general cognitive ability $(r=.48$ to .56$)$. Low CRV, which also indicates successful SL performance, also correlates significantly with general cognitive ability $(r$ $=-.40$ to -.44 ). These results show a substantially stronger link between SL ability and general cognitive function than those previously observed ( $r=0.019$ to .16 in Kaufman et al., 2010 and Feldman et al., 1995). Whilst not speaking to the current debate on domain dependence vs. independence of $\mathrm{SL}$, or shared underlying cognitive mechanisms, the current results suggest that SL ability may be associated in part with general cognitive ability (Ahissar et al., 2006; Conway \& Christiansen, 2005; Conway \& Pisoni, 2008; Frost et al., 2015; Frost et al., 2013; Siegelman \& Frost, 2015).

CHPC and CRV Performance Despite the significant correlations between both SL measurements and cognitive function, the number of participants that exceeded the conservative threshold of chance performance was limited (7 out of 27 during the first session). As a result, the present study faces the same limitation as many other studies investigating SL (see Siegelman, Bogaerts, \& Frost, 2017 for a review), namely, that many participants perform at chance level. The number of participants who performed at chance makes it difficult to develop statistical models to predict the time course of SL, so this activity will be the subject of future research. Siegelman, Bogaerts, and Frost (2017) suggested that one way to avoid chance performance could be to provide participants with a greater number of trials. The current novel SL paradigm was limited to 10 minutes due to time restrictions within the larger study. Most SL studies use substantially more than the 48 trials used here (192 trials in familiarization plus 42 test trials in Siegelman, Bogaerts, \& Frost, 2017, and 960 trials in Kaufman et al., 2010). The fact that some participants were capable of successful SL performance despite the small number of trials is encouraging, and in the future, we hope to extend the current paradigm by increasing the number of trials.

The relatively large number of participants who performed at the conservatively chosen chance level also warrants caution when interpreting the results. For example, the high test-retest reliability observed may be influenced by the fact that the same participants performed above chance in both sessions, thereby potentially inflating the overall correlation values. By modifying the paradigm in the future to allow all participants to perform above chance, we hope to replicate and reassess the test-retest coefficients obtained here. To this end, a multi-modal version of the paradigm may also lead to better results in the future. This is because multimodal presentation may increase the reliability of individual estimates of SL ability, and thereby its validity as a measure of cognitive function, regardless of whether SL is subject to domain dependent constraints or a domain agnostic computational principle (Ahissar et al., 2006; Conway \& Christiansen, 2005; Frost et al., 2015; Frost et al., 2013; Siegelman \& Frost, 2015; Conway \& Pisoni, 2008; Siegelman, Bogaerts, Christiansen, et al., 2017).

\section{Conclusion}

The present study indicates that SL ability can be used to predict general cognitive function as measured by a traditional cognitive assessment battery. Furthermore, SL ability shows high test-retest reliability that is comparable to common psychological assessments. Taken together, the present findings suggest that SL ability may be an underlying computational principle crucial for general cognitive function. The continuous paradigm requires further refinement before it may be used as a reliable measure of cognitive ability in clinical settings. Specifically, we hope to explore multimodal presentation of the stimuli in the future.

\section{References}

Abla, D., Katahira, K., \& Okanoya, K. (2008). On-line assessment of statistical learning by event-related potentials. J Cogn Neurosci, 20(6), 952-964.

Agres, K., Abdallah, S., \& Pearce, M. (2018). InformationTheoretic Properties of Auditory Sequences Dynamically Influence Expectation and Memory. CognSci, 42(1), 43-76.

Ahissar, M., Lubin, Y., Putter-Katz, H., \& Banai, K. (2006). Dyslexia and the failure to form a perceptual anchor. Nature Neuroscience, 9(12), 1558.

Barascud, N., Pearce, M. T., Griffiths, T. D., Friston, K. J., \& Chait, M. (2016). Brain responses in humans reveal ideal observer-like sensitivity to complex acoustic patterns. Proceedings of the National Academy of Sciences, E616E625. doi:10.1073/pnas.1508523113

Conway, C. M., \& Christiansen, M. H. (2005). Modalityconstrained statistical learning of tactile, visual, and auditory sequences. Journal of Experimental Psychology: Learning, Memory, and Cognition, 31(1), 24.

Conway, C. M., \& Pisoni, D. B. (2008). Neurocognitive basis of implicit learning of sequential structure and its relation to language processing. Annals of the New York Academy of Sciences, 1145(1), 113-131.

Creel, S. C., Newport, E. L., \& Aslin, R. N. (2004). Distant melodies: statistical learning of nonadjacent dependencies in tone sequences. Journal of Experimental Psychology: Learning, Memory, and Cognition, 30(5), 1119.

Erickson, L. C., \& Thiessen, E. D. (2015). Statistical learning of language: theory, validity, and predictions of a statistical learning account of language acquisition. Developmental Review, 37, 66-108.

Feldman, J., Kerr, B., \& Streissguth, A. P. (1995). Correlational analyses of procedural and declarative learning performance. Intelligence, 20(1), 87-114.

Frost, R., Armstrong, B. C., Siegelman, N., \& Christiansen, M. H. (2015). Domain generality vs. modality specificity: The paradox of statistical learning Trends Cogn Sci(Mar;19(3)), 117-125. doi:10.1016/j.tics.2014.12.010

Frost, R., Siegelman, N., Narkiss, A., \& Afek, L. (2013). What predicts successful literacy acquisition in a second language? Psychological Science, 24(7), 1243-1252.

Karuza, E. A., Newport, E. L., Aslin, R. N., Starling, S. J., Tivarus, M. E., \& Bavelier, D. (2013). The neural correlates 
of statistical learning in a word segmentation task: An fMRI study. Brain and Language, 127(1), 46-54.

Kaufman, S. B., DeYoung, C. G., Gray, J. R., Jiménez, L., Brown, J., \& Mackintosh, N. (2010). Implicit learning as an ability. Cognition, 116(3), 321-340.

Keefe, R. S. E., Goldberg, T. E., Harvey, P. D., Gold, J. M., Poe, M. P., \& Coughenour, L. (2004). The Brief Assessment of Cognition in Schizophrenia: reliability, sensitivity, and comparison with a standard neurocognitive battery. Schizophrenia Research, 68(2-3), 283-297.

Kirkham, N. Z., Slemmer, J. A., \& Johnson, S. P. (2002). Visual statistical learning in infancy: Evidence for a domain general learning mechanism. Cognition, 83(2), B35-B42.

Lam, M., Collinson, S. L., Eng, G. K., Rapisarda, A., Kraus, M., Lee, J., . . Keefe, R. S. E. (2014). Refining the latent structure of neuropsychological performance in schizophrenia. Psychol Med, 44(16), 3557-3570.

Lam, M., Wang, M., Huang, W., Eng, G. K., Rapisarda, A., Kraus, M., . . . Lee, J. (2017). Establishing the Brief Assessment of Cognition-Short form. Journal of Psychiatric Research, 93, 1-11.

Misyak, J. B., Christiansen, M. H., \& Tomblin, J. B. (2010). On-line individual differences in statistical learning predict language processing. Frontiers in Psychology, 1, 31.

Moldwin, T., Schwartz, O., \& Sussman, E. S. (2017). Statistical Learning of Melodic Patterns Influences the Brain's Response to Wrong Notes. J Cogn Neurosci, 29(12), 2114-2122.

Nunnally, J. C., \& Bernstein, I. H. (1994). Psychometric Theory (McGraw-Hill Series in Psychology) (Vol. 3): McGraw-Hill New York.

Pérez-González, D., \& Malmierca, M. S. (2014). Adaptation in the auditory system: an overview. Frontiers in integrative neuroscience, $8,19$.

Saffran, J. R., Aslin, R. N., \& Newport, E. L. (1996). Statistical learning by 8-month-old infants. Science, 274(5294),1926-1928.doi:10.1126/Science.274.5294.1926

Saffran, J. R., \& Kirkham, N. Z. (2018). Infant statistical learning. Annual Review of Psychology, 69.

Seger, C. A. (1994). Implicit learning. Psychological Bulletin, 115(2), 163.

Siegelman, N., Bogaerts, L., Christiansen, M. H., \& Frost, R. (2017). Towards a theory of individual differences in statistical learning. Phil. Trans. R. Soc. B, 372(1711), 20160059.

Siegelman, N., Bogaerts, L., \& Frost, R. (2017). Measuring individual differences in statistical learning: Current pitfalls and possible solutions. Behavior Research Methods, 49(2), 418-432.

Siegelman, N., \& Frost, R. (2015). Statistical learning as an individual ability: Theoretical perspectives and empirical evidence. Journal of Memory and Language, 81, 105-120.

Unsworth, N., \& Engle, R. W. (2005). Individual differences in working memory capacity and learning: Evidence from the serial reaction time task. Memory \& Cognition, 33(2), 213-220. 